\title{
Interpretation of Abundance Ratios
}

\author{
Francesca Matteucci $^{\mathrm{A}, \mathrm{C}}$ and Cristina Chiappini ${ }^{\mathrm{B}}$ \\ A Dipartimento di Astronomia, Universita' di Trieste, 34127 Trieste, Italy \\ ${ }^{B}$ Osservatorio Astronomico di Trieste (INAF), 34127 Trieste, Italy \\ ${ }^{\mathrm{C}}$ Corresponding author. Email: matteucci@ ts.astro.it
}

Received 2004 April 27, accepted 2004 October 20

\begin{abstract}
In this paper we discuss abundance ratios and their relation to stellar nucleosynthesis and other parameters of chemical evolution models, reviewing and clarifying the correct use of the observed abundance ratios in several astrophysical contexts. In particular, we start from the well-known fact that abundance ratios depend on stellar yields, initial mass function, and stellar lifetimes, and we show, by means of specific examples, that in some cases it is not correct to infer constraints on the contributions from different supernovae types (Ia, II), and particularly on different sets of yields, in the absence of a complete chemical evolution model taking into account stellar lifetimes. In spite of the fact that some of these results should be well known, we believe that it is useful to discuss the meaning of abundance ratios in the light of several recent claims based upon an incorrect interpretation of observed abundance ratios. In particular, the procedure, often used in the recent literature, of directly deriving conclusions about stellar nucleosynthesis just by relating abundance ratios to yield ratios implicitly assumes the instantaneous recycling approximation. This approximation is clearly not correct when one analyses the contributions of supernovae type Ia relative to supernovae type II as functions of cosmic time. In this paper we show that the uncertainty which arises from adopting this oversimplified procedure in a variety of astrophysical objects, such as elliptical galaxies, the intracluster medium, and high redshift objects, does not allow us to draw any firm conclusion, and that the differences between abundance ratios predicted by models with the instantaneous recycling approximation and models with detailed stellar lifetimes is of the same order as the differences between different sets of yields. On the other hand, if one is interested only in establishing the global metal production (e.g. galaxies plus intracluster medium) over the lifetime of the Universe, then the adoption of simplified arguments can be justified.
\end{abstract}

Keywords: nucleosynthesis, abundances — galaxies: evolution

\section{Introduction}

Recently, a great deal of work has been done in measuring chemical abundances not only in stars in the Galaxy but also in the intracluster medium (ICM) and in high-redshift damped-Lyman $\alpha$ (DLA) objects. Therefore, it is challenging for theorists to interpret the meaning of abundance ratios which are less affected than absolute abundances by the assumptions of chemical evolution models, such as star formation rate, infall rate, and outflow rate.

Abundance ratios, in fact, are largely independent of these parameters but depend upon the stellar nucleosynthesis, the initial mass function (IMF), and the stellar lifetimes. On the other hand, the variation of abundance ratios in time or as a function of metallicity depends on the star formation history as well. Because of this, it has often been proposed to use abundance ratios as a function of time or metallicity as cosmic clocks, and in particular the ratios involving one element produced on short timescales and the other produced on large timescales, as for example O/Fe and N/O (Tinsley 1979). The bulk of iron and nitrogen is, in fact, produced on long timescales, whereas oxygen is rapidly produced (on timescales of a few million years) by massive stars. Iron production is due to type Ia supernovae ( $\mathrm{SNe}$ ) which are thought to originate from white dwarfs in binary systems, and is restored over a range of timescales from $30 \mathrm{Myr}$ to $15 \mathrm{Gyr}$ and more. Nitrogen is mainly produced in stars with masses from 2 to $8 M_{\odot}$ and therefore on timescales ranging from several tens of million years to some billion years.

Recent papers discussing the chemical abundances measured in the ICM derive constraints on the particular type Ia SN model just by comparing the measured abundance ratios with stellar production ratios in type II and Ia $\mathrm{SNe}$. In proceeding this way, one implicitly assumes that abundance ratios can give direct information on the production ratios of the elements considered.

In this paper we show that the above assumption can be tolerated only if the abundance ratios concern elements produced on the same timescales, but it is uncertain when applied to ratios such as $\mathrm{O} / \mathrm{Fe}$ involving elements produced on quite different timescales. Moreover, we point out that neglecting the stellar lifetimes can be incorrect even for certain $\alpha$-elements such as $\mathrm{S}$ and $\mathrm{Si}$ when compared to $\mathrm{O}$. This is important in connection with the fact that a 
common procedure is to assume that $\alpha$-elements all evolve in the same way, and in particular to take $\mathrm{O}$ as a proxy for $\mathrm{Si}$ and $\mathrm{S}$ when studying the evolution of DLA systems.

The paper is organised as follows: in Section 2 we discuss how the evolution of the abundance ratios depends on chemical evolution models; in Sections 3, 4, and 5 we discuss the chemical evolution of the solar neighbourhood, ICM, and DLA systems, respectively; finally, in Section 6 we draw some conclusions.

\section{Abundance Ratios and Chemical Evolution of Galaxies}

The Simple Model of chemical evolution assumes that the system studied evolves as a closed box, that the IMF is constant in time, that the gas out of which the first stars form is primordial (i.e. $Z=0$ ), and that the instantaneous recycling approximation (IRA) holds (see Tinsley 1980). This approximation allows us to ignore the stellar lifetimes and therefore the delay with which some of the chemical elements are produced and restored into the ISM. In other words, all the elements are produced instantaneously.

The Simple Model predicts that the abundance of a generic metal $i, X_{i}$, evolves as

$$
X_{i}=y_{i} \ln \mu^{-1}
$$

where $\mu=M_{\mathrm{gas}} / M_{\mathrm{tot}}$ is the gas fraction in the system and $y_{i}$ is the 'yield' per stellar generation:

$$
y_{i}=\frac{1}{(1-R)} \int_{m_{\mathrm{TO}}}^{\infty} m p_{\text {im }} \varphi(m) \mathrm{d} m,
$$

which depends on the $\operatorname{IMF} \varphi(m)$ and the stellar yield $p_{i m}$, namely the fraction of the stellar mass ejected as the newly created element $i$ by a star of mass $m$. The mass $m_{\mathrm{TO}}$ is the globular cluster 'turn-off' mass.

The quantity $R$ is the so-called returned fraction:

$$
R=\int_{m_{\mathrm{TO}}}^{\infty}\left(m-m_{\mathrm{rem}}\right) \varphi(m) \mathrm{d} m
$$

with $m$ being the star mass and $m_{\text {rem }}$ the remnant mass. It is worth noting that both $y_{i}$ and $R$ are fractions since they are both divided by

$$
\int_{m_{\mathrm{TO}}}^{\infty} m \varphi(m) \mathrm{d} m=1
$$

which is the normalisation condition for the IMF. The IMF is generally expressed as

$$
\varphi(m)=A m^{-(1+x)}
$$

where the constant $A$ is derived from equation (4).

If the Simple Model holds, according to equation (1) we can write

$$
\frac{X_{i}}{X_{j}}=\frac{y_{i}}{y_{j}} .
$$

Therefore, in this case the ratio of two abundances is directly the ratio of the two 'yields' defined in equation (2).
The same is true if instead of the Simple Model we consider models with inflows/outflows but always with IRA approximation.

For example, in the presence of outflow and under the assumption that the outflow rate is simply proportional to the star formation rate multiplied by $(1-R)$ through a constant $\lambda$, one can find the following solution for metals (see Matteucci \& Chiosi 1983; Matteucci 2001):

$$
X_{i}=\frac{y_{i}}{(1-\lambda)} \ln \left[(1+\lambda) \mu^{-1}-\lambda\right]
$$

where $\lambda$ is a constant larger than zero. Again, the abundance ratio of the two elements coincides with the ratio of their yields.

A similar situation occurs for an infall model with IRA when there is infall of primordial material without metals $\left[\left(X_{i}\right)_{\text {inf }}=0\right]$ and the infall rate is proportional to the star formation rate multiplied by $(1-R)$ through a positive constant $\Lambda \neq 1$ (Matteucci \& Chiosi 1983; Matteucci 2001):

$$
X_{i}=\frac{y_{i}}{\Lambda}\left\{1-\left[\Lambda-(\Lambda-1) \mu^{-1}\right]^{[-\Lambda /(1-\Lambda)]}\right\}
$$

If $\Lambda=1$ the solution, always for metals, is

$$
X_{i}=y_{i}\left[1-\mathrm{e}^{-\left(\mu^{-1}-1\right)}\right]
$$

which is the well-known solution for extreme infall (Larson 1972), where the amount of gas in the system remains constant in time.

Finally, in the case of infall and outflow operating at the same time the solution is:

$X_{i}=\frac{y_{i}}{\Lambda}\left\{1-\left[(\Lambda-\lambda)-(\Lambda-\lambda-1) \mu^{-1}\right]^{[\Lambda /(\Lambda-\lambda-1)]}\right\}$,

for a primordial infalling gas $\left[\left(X_{i}\right)_{\mathrm{inf}}=0\right]$ as shown in Matteucci (2001).

In all of these cases (eqns 7-10) it is evident that equation (6) holds, but this is not true anymore if one relaxes IRA, as is shown in Figure 1 where we plot the predicted $\mathrm{O} / \mathrm{Fe}$ abundance ratio (abundances by mass) as a function of time, as predicted by a detailed model of the Milky Way (Chiappini, Romano, \& Matteucci 2003). As one can see, in fact, the $[\mathrm{O} / \mathrm{Fe}]$ ratio is strongly varying with time and this is due to the delayed $\mathrm{Fe}$ production relative to the $\mathrm{O}$ production.

This delay allows us to interpret $[\mathrm{O} / \mathrm{Fe}]$ versus $[\mathrm{Fe} / \mathrm{H}]$ observed for solar neighbourhood stars (Greggio \& Renzini 1983; Matteucci \& Greggio 1986). In the same figure we show the constant $\mathrm{O} / \mathrm{Fe}$ production ratio expected from a typical SNII and a typical SNIa (dashed and dotdashed lines, respectively). In particular, we took the production ratio of a $20 M_{\odot}$ star, as representative of a typical massive star, from Nomoto et al. (1997).

On the other hand, for the ratios between elements produced on similar timescales, such as the ratios between different $\alpha$-elements which are mainly originating from massive short-lived stars, one can reasonably assume that they are almost constant during the whole galactic lifetime. 


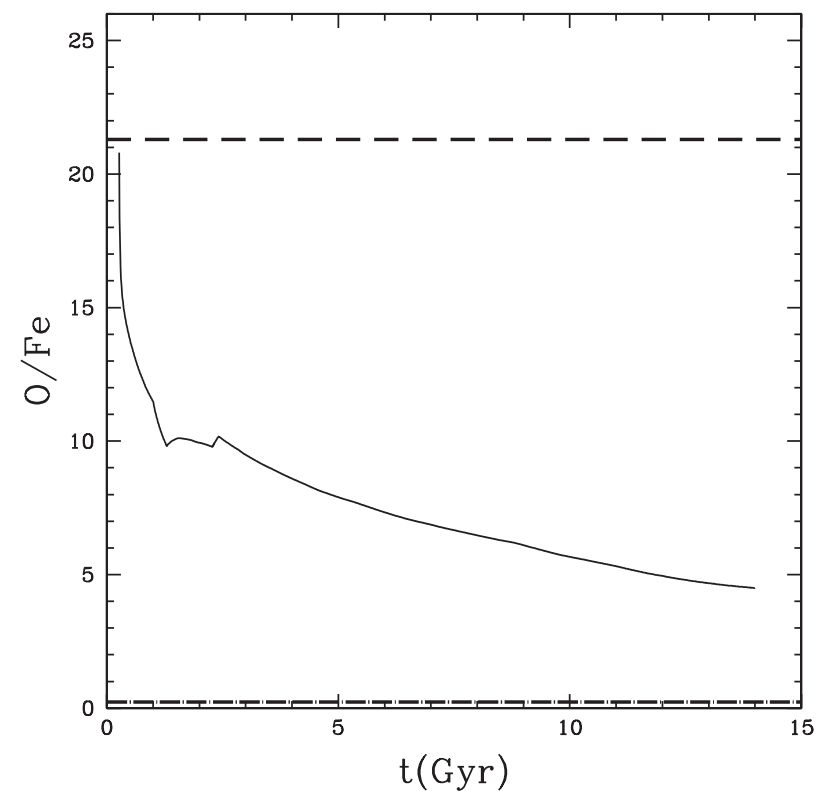

Figure 1 Predicted $\mathrm{O} / \mathrm{Fe}$ abundance ratio (abundance by mass) as a function of time, as predicted by the time-delay model of chemical evolution for the solar vicinity (Chiappini et al. 2003). Also shown are the $(\mathrm{O} / \mathrm{Fe})_{\mathrm{SNII}}$ (dashed line) and $(\mathrm{O} / \mathrm{Fe})_{\mathrm{SNIa}}$ (dash-dotted line) production ratios as given in Nomoto et al. (1997) both for a type Ia SN and a $20 M_{\odot}$ star taken as representative of an average massive star exploding as a type II SN.

Therefore, the assumption that abundance ratios reflect stellar yields is true to a first approximation only for elements produced on the same timescales, such as $\alpha$ elements, although also in this case there are exceptions, as we will show in Section 5.

\section{The Roles of $\mathrm{SNe}$ in the $[\mathrm{O} / \mathrm{Fe}]$ versus $[\mathrm{Fe} / \mathrm{H}] \mathrm{Plot}$ for the Milky Way}

In Figure 2 we show the $[\mathrm{O} / \mathrm{Fe}]$ versus $[\mathrm{Fe} / \mathrm{H}]$ relation as predicted by the best model for the solar neighbourhood of Chiappini et al. (2003) (model 4 with yields of Nomoto et al. 1997). The model reproduces very well the most recent and accurate measurements in stars of the solar neighbourhood. The model is a two-infall model where the halo-thick disk forms on a relatively short timescale $(\sim 1-$ $2 \mathrm{Gyr}$ ) whereas the disk forms on much longer timescales and in an 'inside-out' fashion. The best model takes into account detailed nucleosynthesis from types II and Ia SNe, and predicts that type Ia SNe produce $\sim 70 \%$ of the present Fe abundances, whereas the remaining 30\% comes from type II SNe. This prediction is a direct consequence of the assumed stellar yields and IMF. The yields of $\mathrm{O}$ and $\mathrm{Fe}$ from massive stars and the yields of Fe from type Ia $\mathrm{SNe}$ are from Nomoto et al. (1997), their model W7, while for the IMF we assume that of Scalo (1986). On the same Figure we show one model where we arbitrarily assumed that all the Fe would arise from type II SNe and one model where all the $\mathrm{Fe}$ originates from type Ia SNe. All the models and the data are normalised to the solar values of Anders \& Grevesse (1989). As one can see, neither of the two models would fit the observed pattern since Fe

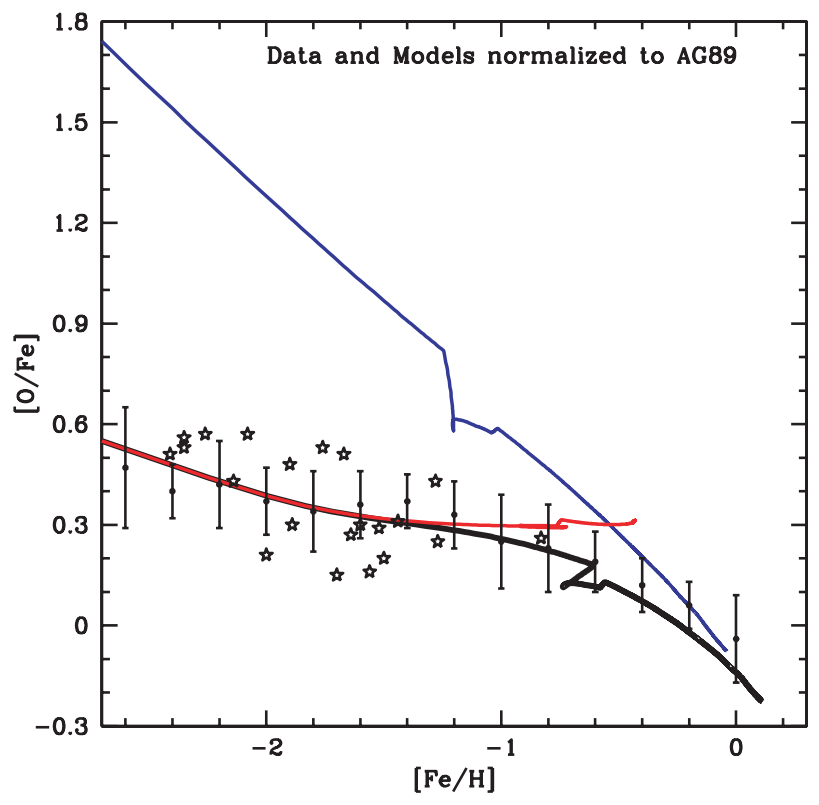

Figure 2 Predicted $[\mathrm{O} / \mathrm{Fe}]$ versus $[\mathrm{Fe} / \mathrm{H}]$ from the model of Chiappini et al. (2003) (thick continuous line), compared with the data from Meléndez \& Barbuy (2002). The strongly decreasing line represents the prediction of the model when the Fe production from type II SNe is suppressed, whereas the almost constant line refers to the predictions when the $\mathrm{Fe}$ production from type $\mathrm{Ia} \mathrm{SNe}$ is suppressed.

originating only from type II SNe would produce a flat $[\mathrm{O} / \mathrm{Fe}]$ over the entire $[\mathrm{Fe} / \mathrm{H}]$ range, as expected. On the other hand, if all the Fe were to originate from type Ia $\mathrm{SNe}$ then the $[\mathrm{O} / \mathrm{Fe}]$ would be linearly decreasing with increasing metallicity without showing any plateau at low metallicities. Clearly, to explain the observed pattern we need both SN types and, to do that, we need to relax the IRA approximation. For this reason, this kind of model is often referred to as a time-delay model. In fact, under IRA we would always predict a constant $[\mathrm{O} / \mathrm{Fe}]$ for the whole metallicity range. Therefore, in conclusion, ignoring the effect of stellar timescales produces an incorrect interpretation of data of any kind.

Intuitively, one may think that after a very long time since the end of star formation, when the gas content tends to zero, the abundance ratios will tend to the ratios of their yields per stellar generation (equation 2). This is true, in principle, if the global metal production is considered (namely the metals in stars, and in gas inside and outside galaxies), but it fails if only the metals in the individual components (e.g. in the gas) are taken into account. In fact, as shown by Prantzos \& Aubert (1995), just when the gas in a system tends to zero then the effects of relaxing IRA are the strongest. In particular, the final amount of gas in the system is influenced by relaxing IRA, since low mass stars restore their external envelopes at this time and these envelopes contain the abundance patterns of the gas in the early stages of galactic evolution. The effect of this delayed gas return is negligible in galaxies with ongoing star formation and therefore possessing a relatively high fraction of gas, whereas it is important in objects 
which have stopped forming stars several Gyr ago, such as ellipticals, as we will show in the next Section.

\section{Abundances in the ICM}

Several papers have already appeared in the literature trying to interpret the measured abundance ratios in the ICM simply in terms of stellar yields, with the consequence of imposing constraints on different nucleosynthesis models for type Ia SNe as well as on the different proportions of Fe produced by type Ia and II SNe (see for example Gibson, Lowenstein, \& Mushotzky 1997; Finoguenov et al. 2002; Gastaldello \& Molendi 2002). For instance, the empirical method adopted in the observational papers dealing with ICM abundance ratios can be summarised as follows: the observed ratios of several elements to iron are used in order to find the best fit to a function defined as

$$
\begin{aligned}
\left(\frac{X / \mathrm{Fe}}{X_{\odot} / \mathrm{Fe}_{\odot}}\right)_{\text {observed }}= & f\left(\frac{X / \mathrm{Fe}}{X_{\odot} / \mathrm{Fe}_{\odot}}\right)_{\mathrm{SNIa}} \\
& +(1-f)\left(\frac{X / \mathrm{Fe}}{X_{\odot} / \mathrm{Fe}_{\odot}}\right)_{\mathrm{SNII}},
\end{aligned}
$$

where the ratios labelled SNIa and SNII are the ratios of the stellar yields expected from SNIa and SNII relative to the solar abundance ratios, respectively. The quantity $f$ represents the unknown fraction of Fe produced by SNIa relative to the total $\mathrm{Fe}$ produced by type Ia plus type II $\mathrm{SNe}$. In principle, equation (11) is valid if one is interested in the global metal production (stars, gas inside and outside galaxies), but it provides a poor approximation if one studies the abundances in the different components individually, such as the abundances in the ICM and especially their evolution with cosmic time. One reason for this resides in the fact that IRA is a bad approximation for studying the evolution of the Fe abundance.

Moreover, in the approach described by equation (11), what is completely ignored is the mechanism of chemical enrichment of the ICM from galaxies which strongly affects the ICM abundance ratios. Several steps are necessary to compute the ICM chemical abundances. The first one is to compute the chemical evolution of elliptical galaxies. Elliptical galaxies are, in fact, believed to be the main contributors to the ICM enrichment (e.g. Matteucci \& Vettolani 1988; Arnaud et al. 1992; Matteucci \& Gibson 1995; Renzini 1997; Chiosi 2000; Pipino et al. 2002; Moretti, Portinari, \& Chiosi 2003). In Figure 3 we show the evolution of the ratio of a typical $\alpha$-element such as $\mathrm{O}$ relative to $\mathrm{Fe}$ as a function of time, as predicted for the gas in elliptical galaxies of luminous mass $\sim 10^{11}$ and $\sim 10^{10} M_{\odot}$. In the same figure is shown the constant $\mathrm{O} / \mathrm{Fe}$ ratio derived from the ratio of the yields (production ratios) of oxygen and iron (dashed line for SNII, and dot-dashed line for SNIa). As one can clearly see from the figure, the $\mathrm{O} / \mathrm{Fe}$ ratios in the gas in ellipticals, after the star formation stops, are not constant but they first decrease, as expected because of the occurrence of more and more SNIa, then increase and finally reach a plateau at late times. This latter effect, often forgotten, is due to

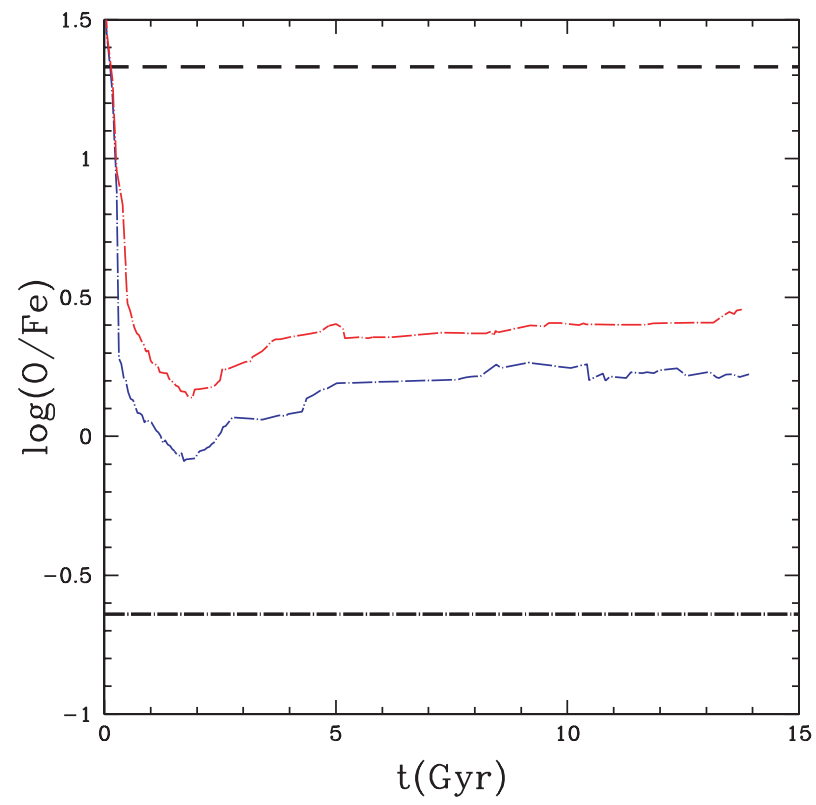

Figure 3 Predicted $\log (\mathrm{O} / \mathrm{Fe})$ versus time in the ISM of elliptical galaxies before and after the occurrence of galactic winds which start at early times $(\leq 0.3 \mathrm{Gyr})$ and continue until the present time. The model predictions are from Pipino et al. (2002), their Model I, inverse wind case, with Salpeter (1955) IMF. The galaxies have masses of $10^{10} M_{\odot}$ (lower curve) and of $10^{11} M_{\odot}$ (upper curve). The abundances are by mass. The constant lines represent the $\mathrm{O} / \mathrm{Fe}$ yields for massive stars (dashed line) and for type Ia SNe (dash-dotted line) from Nomoto et al. (1997, their W7 model for type Ia SNe), as in Figure 1.

the relaxation of IRA and is produced by the low mass stars formed out of gas enriched in oxygen relative to iron at early times, as already mentioned. These stars, in fact, eject through the planetary nebula phase their pristine gas with a high $\mathrm{O} / \mathrm{Fe}$ ratio and this gas can be restored later on into the ICM by means of galactic winds and/or ram pressure stripping. This is the case of Figure 3 since the models presented here predict a continuous galactic wind since early times. In particular, the galactic winds start before $0.3 \mathrm{Gyr}$ in both galaxies, thus most of the Fe produced by type Ia SNe is ejected into the ICM. It is worth noting that the final $\mathrm{O} / \mathrm{Fe}$ ratios contributed by galaxies of different luminous masses $\left(10^{10}, 10^{11} M_{\odot}\right)$ are different and the difference depends on the duration of the star formation period in each galaxy. This occurs because the degree of $\alpha$-enhancement depends on the timescale of the Fe released by type Ia SNe relative to the timescale of star formation (see Matteucci \& Recchi 2001). Good models of chemical evolution for elliptical galaxies predict that the star formation timescale was shorter in more massive objects, in order to reproduce the observed increase of the $[\alpha / \mathrm{Fe}]$ ratio in the dominant stellar population as a function of galactic mass (Matteucci 1994; Pipino et al. 2002; Pipino \& Matteucci 2004; Romano et al. 2003). This implies that galactic winds should occur first in massive objects, thus interrupting the star formation process. As a consequence of this, the final $\log (\mathrm{O} / \mathrm{Fe})$ produced by a $10^{10} M_{\odot}$ (luminous mass) galaxy is $\sim 0.2$ whereas 
that produced by a $10^{11} M_{\odot}$ galaxy is $\sim 0.5$. The difference, $\Delta \log (\mathrm{O} / \mathrm{Fe}) \sim 0.3$, is comparable to the differences among production ratios obtained by adopting different sets of yields. For example, if we take the SNII oxygen yield and the yields of Fe predicted by the different type Ia models of Iwamoto et al. (1999) we find that the maximum difference between production ratios is $\Delta \log (\mathrm{O} / \mathrm{Fe}) \sim 0.7$ (between model WDD3 and model CDD1 which produce the highest and lowest $\mathrm{Fe}$ in type Ia $\mathrm{SNe}$, respectively).

In realistic models for the chemical enrichment of the ICM (e.g. Matteucci \& Vettolani 1988; Matteucci \& Gibson 1995; Pipino et al. 2002), one should integrate the contribution of single cluster galaxies to the $\mathrm{O}$ and Fe enrichment in the ICM by means of the cluster luminosity function. The total masses of $\mathrm{O}$ and $\mathrm{Fe}$ which are ejected into the ICM crucially depend on the assumptions about the duration of galactic winds and/or ram pressure stripping, in other words about how much mass is lost from the galaxies (generally the mass at the break of the luminosity function dominates the ICM enrichment). In particular, in Matteucci \& Vettolani (1988) and Pipino et al. (2002) it was found that the Fe mass observed in galaxy clusters, either poor or rich, can be reproduced with a Salpeter (1955) IMF, only if all the Fe produced by type Ia $\mathrm{SNe}$, after the star formation has stopped, is sooner or later restored into the ICM. On the other hand, a top-heavy or a variable IMF (Matteucci \& Gibson 1995; Chiosi 2000; Moretti et al. 2003) can reproduce all the observed ICM Fe with the only contribution from early galactic winds.

A top-heavy IMF rather than a Salpeter-like IMF increases the $[\alpha / \mathrm{Fe}]$ ratio predicted for the dominant stellar population in ellipticals, but it does not prohibit obtaining an almost solar $[\alpha / \mathrm{Fe}]$ ratio in the ICM at the present time, if one assumes that all the Fe produced by type Ia $\mathrm{SNe}$, after star formation ceases, sooner or later will be restored into the ICM; then, however, the total Fe mass restored into the ICM is overestimated. In this case, one is forced to assume that most of the Fe produced by type Ia $\mathrm{SNe}$ has been retained by the galactic potential well and has not been mixed with the ICM. As a consequence of this, one predicts an overabundance of oxygen relative to iron $([\alpha / \mathrm{Fe}]>0)$ in the local ICM.

Therefore, it is clear that, because of all the effects mentioned above, the particular effect of the stellar yields on the $\mathrm{O} / \mathrm{Fe}$ ratio in the ICM is quite difficult to extract from the measured abundance ratios. As a consequence, no firm conclusion about the different nucleosynthesis models for type Ia $\mathrm{SNe}$ can be drawn on the basis of the simple assumption that abundance ratios are indicative of the yield ratios.

What is instead a robust conclusion is that if the observed $[\alpha / \mathrm{Fe}]$ ratio in the ICM is solar or slightly undersolar (although the measured abundance ratios in clusters are still uncertain and do not allow us to draw firm conclusions; see Loewenstein 2004 for a recent review on the subject), one can safely conclude that the contribution of type Ia SNe to the Fe enrichment, relative to the
Fe enrichment from type II SNe, has been the same as in the solar vicinity. In fact, if the $[\alpha / \mathrm{Fe}]$ enhancement observed in the Galactic halo stars is interpreted as being due to the prompt enrichment by type II SNe and that the almost solar $[\alpha / \mathrm{Fe}]$ seen in the Galactic disk stars is due to the addition of Fe from type Ia SNe (see Figure 2), then the same reasoning should hold for the ICM (Renzini 1997), irrespective of the differences among different sets of yields. This is at variance with the conclusion of Gibson et al. (1997) who stated that, owing to the uncertainties still present in the stellar yields, no firm conclusions on the role of type Ia and II SNe in the ICM enrichment can be drawn. In this case, in fact, only those yields should be used which best reproduce $[\alpha / \mathrm{Fe}]$ versus $[\mathrm{Fe} / \mathrm{H}]$ in the solar neighbourhood, given the supposed universality of nucleosynthesis.

\section{Damped Lyman- $\alpha$ Systems}

Another important application of the abundance ratios is to infer the nature and the age of DLAs, namely the absorbers of quasar light observed at high redshift. In particular, these objects possess a high neutral gas content $\left(N_{\mathrm{HI}} \geq 2 \times 10^{20} \mathrm{~cm}^{-2}\right)$ and metal abundances ranging from $\sim 1 / 100$ up to $\sim 1 / 3$ of the solar value (from $[\mathrm{Fe} / \mathrm{H}]$ $>-2.0 \mathrm{dex}$ ). Since different histories of star formation in different galaxies produce different $[\alpha / \mathrm{Fe}]$ versus $[\mathrm{Fe} / \mathrm{H}]$ relations, as first pointed out by Matteucci (1991) and as shown in Figure 4, one can try to infer the nature of DLA systems by comparing the abundance patterns with galactic chemical evolution models (see Calura, Matteucci, \& Vladilo 2003). In Figure 4 we show the $[\alpha / \mathrm{Fe}]$ versus $[\mathrm{Fe} / \mathrm{H}]$ (where $\alpha$ indicates $\mathrm{O}+\mathrm{Mg}$ ) relations predicted for different histories of star formation: For a system with an intense star formation rate, such as the galactic Bulge, the predicted $[\alpha / \mathrm{Fe}]$ stays constant for a longer $[\mathrm{Fe} / \mathrm{H}]$ interval than in the solar vicinity with less star formation and in an irregular Magellanic system with even less star formation.

The different behaviour of the $[\alpha / \mathrm{Fe}]$ ratio can easily be understood on the basis of the time delay model described in Section 3. A comparison with observed abundance ratios in DLA systems indicates that these objects are likely to be irregular galaxies rather than spheroids such as bulges and elliptical galaxies. Therefore the $[\alpha / \mathrm{Fe}]$ versus $[\mathrm{Fe} / \mathrm{H}]$ ratios represent a unique diagnostic for revealing the nature of high redshift objects.

Often in the literature (Pettini et al. 2002; Prochaska et al. 2002; Centurión et al. 2003), when dealing with DLA systems, $\mathrm{S}$ and $\mathrm{Si}$ are used as proxies for $\mathrm{O}$, because they are all $\alpha$-elements. However, also among $\alpha$-elements there are differences, in particular between $\mathrm{O}$ on the one hand and $\mathrm{S}$ and $\mathrm{Si}$ on the other. These latter elements, in fact, according to the majority of the available stellar yields, are produced in a non-negligible way by $\mathrm{SNe}$ Ia, whereas oxygen is entirely produced by massive stars. This difference is reflected in the predicted $\mathrm{O} / \mathrm{S}$ and $\mathrm{O} / \mathrm{Si}$ ratios, as shown in Figure 5. There it is clear that the $\mathrm{O} / \mathrm{S}$ and $\mathrm{O} / \mathrm{Si}$ ratios predicted for the solar neighbourhood are not constant as functions of $[\mathrm{Fe} / \mathrm{H}]$ and therefore time, as one 


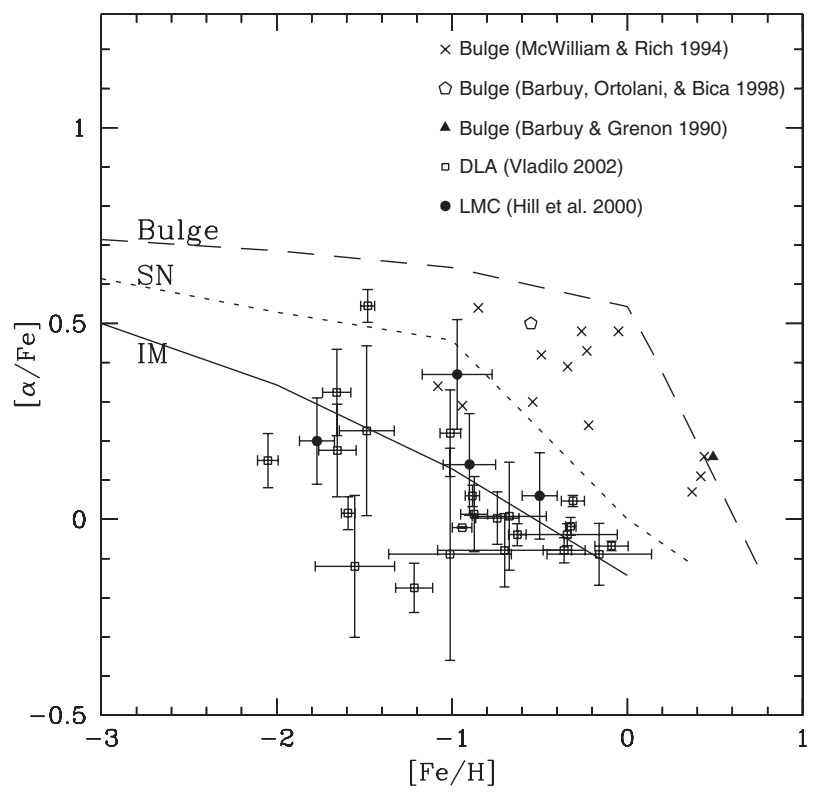

Figure 4 Predicted $[\alpha / \mathrm{Fe}]$ versus $[\mathrm{Fe} / \mathrm{H}]$ for different histories of star formation. In particular, the upper curve represents the galactic Bulge, the central one the solar vicinity, and the lower one an irregular Magellanic galaxy. The data refer to the Bulge stars and to DLA systems, as explained in the text.

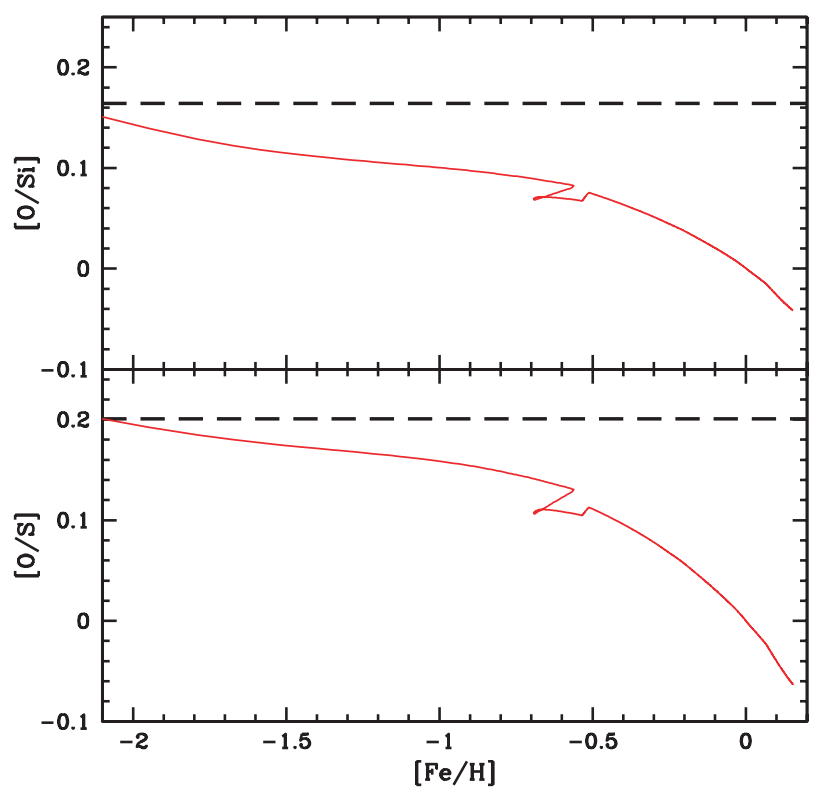

Figure 5 Predicted $[\mathrm{O} / \mathrm{S}]$ and $[\mathrm{O} / \mathrm{Si}]$ as functions of $[\mathrm{Fe} / \mathrm{H}]$ by the model of Chiappini et al. (2003) for the solar vicinity. The constant lines represent the $(\mathrm{O} / \mathrm{Si})_{\text {yields }}$ and $(\mathrm{O} / \mathrm{S})_{\text {yields }}$ ratios for massive stars taken from Nomoto et al. (1997).

would expect if $\mathrm{S}$ and $\mathrm{Si}$ were proxies for oxygen. The variations of the predicted $\mathrm{O} / \mathrm{S}$ and $\mathrm{O} / \mathrm{Si}$ abundance ratios are partly due to the mass dependence of their production ratios in massive stars and partly to the fact that $\mathrm{S}$ and $\mathrm{Si}$ are produced in type Ia SNe more than is oxygen. We recall that the very first $\mathrm{SNe} \mathrm{Ia}$, in the framework of the progenitor model adopted here (single degenerate scenario, see Matteucci \& Recchi 2001), occur already after $\sim 30-40$ Myr since the beginning of star formation. The deviation of the predicted $\mathrm{O} / \mathrm{S}$ and $\mathrm{O} / \mathrm{Si}$ abundance ratios from the corresponding production ratios in a typical massive star, as shown in Figure 5, is non-negligible especially in the observed range of DLA systems.

Therefore, there is a danger in interpreting abundance ratios relative to $\mathrm{Si}$ and $\mathrm{S}$ as if they were ratios relative to oxygen.

\section{Conclusions}

In this paper we discussed the correct use of abundance ratios in order to infer valuable constraints on the stellar nucleosynthesis and the star formation history in galaxies. In particular, we have pointed out that:

- Abundance ratios depend not only on stellar yields and the IMF but also on the timescales of production of the various elements. This means that, when relaxing the hypothesis of instantaneous recycling, the abundance ratios are not good indicators of yield ratios, as is often assumed in the current literature.

- Observed abundance ratios at the present time in galaxies with a small gas content are affected by the often ignored effect of late gas pollution due to low mass stars restoring, at the present time, the $\mathrm{H}$-rich and $\alpha / \mathrm{Fe}-$ enhanced gas out of which they formed at early times. We have calculated the specific case of elliptical galaxies which are believed to have stopped forming stars in a substantial way several Gyr ago, and have shown that the predicted present time abundance ratios (e.g. $\alpha / \mathrm{Fe}$ ) do not reflect the yield ratios (we adopted the same yields and IMF) but vary according to the initial luminous galactic mass which influences the history of star formation and galactic winds in each object. In particular, the differences between the abundance ratios of ellipticals of different mass are comparable to the differences among different sets of yields. As a consequence, it is a risky procedure to impose constraints upon different sets of yields for SN II and Ia directly from the observed abundance ratios, especially from the abundance ratios measured in the ICM. A correct interpretation of these abundances requires a detailed galactic model able to follow the evolution of the absolute abundances in time plus specific assumptions about the amount of gas ejected into the ICM by ellipticals. The direct comparison of abundance ratios with the yields per stellar generation is, in principle, a valid procedure if one is interested only in computing the global metal production, including gas and stars in galaxies plus the ICM, over the lifetime of the Universe (see Calura \& Matteucci 2004 for a detailed calculation of the global metal production in galaxies plus ICM/IGM).

- As a consequence of the previous point, the observed $[\alpha / \mathrm{Fe}]$ ratio in the ICM depends not only on the yield ratios but also on the star formation timescales in ellipticals. These in turn are related to the time of occurrence of a galactic wind, and to the question of whether all the $\mathrm{Fe}$ produced by type Ia $\mathrm{SNe}$, after star formation 
has stopped, is sooner or later restored into the ICM or is partly retained by the galactic potential well. Unfortunately, the observed abundance ratios in the ICM are still too uncertain to draw firm conclusions, such as suggesting particular IMFs for galaxy clusters, and in any case it would be better to consider the global metal production including galaxies plus ICM (see Portinari et al. 2004 for a discussion of these issues).

- Also, the ratios of different $\alpha$-elements, especially $\mathrm{O}$ on the one hand and $\mathrm{Si}$ and $\mathrm{S}$ on the other, are not constant in time, as expected in a first approximation, partly because $\mathrm{O} / \mathrm{Si}$ and $\mathrm{O} / \mathrm{S}$ production ratios are not constant in massive stars, but mainly because $\mathrm{Si}$ and $\mathrm{S}$ are produced in a non-negligible way also by longlived type Ia SNe. For this reason, $\mathrm{Si}$ and $\mathrm{S}$ cannot be used safely as proxies for $\mathrm{O}$, as is often assumed in interpreting data for DLA systems, since the variation of the $[\mathrm{O} / \mathrm{Si}]$ and $[\mathrm{O} / \mathrm{S}]$ ratios over the metallicity range typical of DLAs can be as high as $\sim 0.3$ and $\sim 0.2$ dex, respectively.

\section{Acknowledgments}

We are indebted to S. Recchi, F. Calura, and A. Pipino for their invaluable help. We also thank an anonymous referee for her/his careful reading and very useful suggestions. We acknowledge financial support from the COFIN2003 project (MIUR) Prot. N.2003028039.

\section{References}

Anders, E., \& Grevesse, N. 1989, GeCoA, 53, 197

Arnaud, M., Hughes, J. P., Forman, W., Jones, C., Lachieze-Rey, M., Yamashita, K., \& Hatsukade, I. 1992, ApJ, 390, 345

Barbuy, B., \& Grenon, M. 1990, in ESO/CTIO Workshop, Bulges of Galaxies, eds. B. J. Jarvis, \& D. M. Terndrup (Garching: ESO Publications), 83

Barbuy, B., Ortolani, S., \& Bica, E. 1998, A\&AS, 132, 333

Calura, F., \& Matteucci, F. 2004, MNRAS, 350, 351

Calura, F., Matteucci, F., \& Vladilo, G. 2003, MNRAS, 340, 59

Centurión, M., Molaro, P., Vladilo, G., Péroux, C., Levshakov, S. A., \& D'Odorico, V. 2003, A\&A, 403, 55

Chiappini, C., Romano, D., \& Matteucci, F. 2003, MNRAS, 339, 63

Chiosi, C. 2000, A\&A, 364, 423
Finoguenov, A., Matsushita, K., Böhringer, H., Ikebe, Y., \& Arnaud, M. 2002, A\&A, 381, 21

Gastaldello, F., \& Molendi, S. 2002, ApJ, 572, 160

Gibson, B. K., Loewenstein, M., \& Mushotzky, R. F. 1997, MNRAS, 290,623

Greggio, L., \& Renzini, A. 1983, MmSAI, 54, 311

Hill, V., François, P., Spite, M., Primas, F., \& Spite, F. 2000, A\&A, 364, 1

Iwamoto, K., Brachwitz, F., Nomoto, K., Kishimoto, N., Umeda, H., Hix, W. R., \& Thielemann, F.-K. 1999, ApJS, 125, 439

Larson, R. B. 1972, NPhS, 236, 7

Loewenstein, M. 2004, in Carnegie Observatories Astrophysics Series, Vol. 4: Origin and Evolution of the Elements, eds. A. McWilliam, \& M. Rauch (Cambridge: Cambridge University Press), 425

Matteucci, F. 1991, in SN 1987A and Other Supernovae, eds. I. J. Danziger, \& K. Kjär (Garching: ESO), 703

Matteucci, F. 1994, A\&A, 288, 57

Matteucci, F. 2001, The Chemical Evolution of the Galaxy (Dordrecht: Kluwer)

Matteucci, F., \& Chiosi, C. 1983, A\&A, 123, 121

Matteucci, F., \& Gibson, B. K. 1995, A\&A, 304, 11

Matteucci, F., \& Greggio, L. 1986, A\&A, 154, 279

Matteucci, F., \& Recchi, S. 2001, ApJ, 558, 351

Matteucci, F., \& Vettolani, G. 1988, A\&A, 202, 21

McWilliam, A., \& Rich, R. M. 1994, ApJS, 91, 749

Meléndez, J., \& Barbuy, B. 2002, ApJ, 575, 474

Moretti, A., Portinari, L., \& Chiosi, C. 2003, A\&A, 408, 431

Nomoto, K., Iwamoto, K., Nakasato, N., Thielemann, F.-K., Brachwitz, F., Tsujimoto, T., Kubo, Y., \& Kishimoto, N. 1997, $\mathrm{NuPhA}, 621,467$

Prantzos, N., \& Aubert, O. 1995, A\&A, 302, 69

Pettini, M., Ellison, S. L., Bergeron, J., \& Petitjean, P. 2002, A\&A, 391, 21

Pipino, A., \& Matteucci, F. 2004, MNRAS, 347, 968

Pipino, A., Matteucci, F., Borgani, S., \& Biviano, A. 2002, NewA, 7, 227

Portinari, L., Moretti, A., Chiosi, C., \& Sommer-Larsen, J. 2004, ApJ, 604, 579

Prochaska, J. X., Henry, R. B. C., O’Meara, J. M., Tytler, D., Wolfe, A. M., Kirkman, D., Lubin, D., \& Suzuki, N. 2002, PASP, 114,993

Renzini, A. 1997, ApJ, 488, 35

Romano, D., Silva, L., Matteucci, F., \& Danese, L. 2003, MNRAS, 346, 295

Salpeter, E. E. 1955, ApJ, 121, 161

Scalo, J. M. 1986, FCPh, 11, 1

Tinsley, B. M. 1979, ApJ, 229, 1046

Tinsley, B. M. 1980, FCPh, 5, 287

Vladilo, G. 2002, A\&A, 391, 407 